$6.68-12.13 \mathrm{ng} / \mathrm{ml})(\mathrm{p}=0.002$ and $\mathrm{p}=0.001$, respectively). Serum Slit2 was elevated in SSc patients irrespective of the nailfold videocapillaroscopy (NVC) pattern or the presence/absence of digital ulcers. Interestingly, differences in serum Slit2 levels were found between VEDOSS patients with early/active NVC pattern and controls $(p<0.0005)$, while Slit2 concentrations were similar in VEDOSS with normal NVC and controls. In SSc, Slit2 and Robo4 expression was higher in clinically affected skin and cultured MVECs in respect to controls. No difference was found in Robo1 expression. Cell viability, wound healing capacity and capillarylike tube formation in H-MVECs were all significantly reduced after challenge with rh Slit2 or SSc sera. These inhibitory effects were significantly attenuated when SSc sera were preincubated with anti-Slit2 blocking antibody. Cell viability, wound healing capacity and in vitro angiogenesis were severely compromised in SScMVECs and could be significantly ameliorated by Slit2 neutralisation or Robo4 gene silencing.

Conclusions: In SSc, increased circulating levels of Slit2 and activation of the Slit2/Robo4 antiangiogenic axis may contribute to peripheral microangiopathy since the very early phase of the disease.

Disclosure of Interest: None declared

DOI: 10.1136/annrheumdis-2018-eular.4610

\section{OP0095 SOLUBLE CD163 AS A POTENTIAL BIOMARKER IN SYSTEMIC SCLEROSIS}

C. Frantz, S. Pezet, J. Avouac, Y. Allanore. INSERM U1016, UMR8104, Paris

Descartes University, Rheumatology A department, Cochin Hospital, Paris, France

Background: Recent accumulating evidences indicate a crucial role of macrophage lineage in the pathogenesis of fibrotic diseases including systemic sclerosis (SSc). CD163 is a surface marker expressed by M2 macrophages that accumulate during the healing phase of acute inflammation. It is actively released from the plasma membrane in response to certain inflammatory stimuli and enters the circulation in its soluble form (sCD163).

Objectives: In this study, we aimed to evaluate the performance of serum and urinary sCD163 concentrations as possible biomarker in SSc.

Methods: Urine and serum samples were obtained from SSc patients, fulfilling the 2013 ACR/EULAR classification criteria for SSc, and age- and sex-matched controls. Serum and urinary SCD163 concentrations were measured by commercially available ELISA kit ( $R$ and $D$ systems) and evaluated for their significance as potential biomarkers. Statistical analysis was carried out using Mann-Whitney $U$ test and the relationship between parameters was statistically examined by Spearman's rank test.

Results: Two hundred and three SSc patients were included, $163(80 \%)$ were female, with a mean \pm standard deviation (SD) age of $59 \pm 13$ years and a mean $\pm S D$ disease duration of $12 \pm 9$ years. Eighty-one (41\%) patients had diffuse cutaneous SSc and mean \pm SD mRSS was 6.6 \pm 7.7 . Lung fibrosis on imaging was observed in $33 \%$ of the patients, $7 \%$ had pulmonary arterial hypertension, $44 \%$ had history of digital ulcers and $41 \%$ were taking immunosuppressive therapy. Control group consisted of 47 age- and sex-matched patients with non-inflammatory diseases, being osteoporosis for the very large majority.

Serum SCD163 levels were significantly higher in SSc patients compared with controls (mean $\pm S D$ : $529 \pm 251$ vs $385.1 \pm 153 \mathrm{ng} / \mathrm{ml}$; $<0.001$ ). Urinary sCD163 concentrations in SSc patients were also higher than those in controls, but this did not reach significance $(236.9 \pm 498$ vs $176.2 \pm 173 \mathrm{ng} / \mathrm{mg} \mathrm{uCr} ; \mathrm{p}=0.580)$. When looking at the subsets according to skin disease or disease duration, no difference could be identified. Furthermore, when the organ involvements were investigated, no subpopulation could be identified as having higher concentrations.

Conclusions: To our knowledge this is the first evaluation of both serum and urinary SCD163 levels in SSc. Our results show a significant difference for sera values that should be prioritised for further studies as compared to urinary concentrations conversely to what has been described in lupus. Our results further support that the M2 macrophages/CD163 signalling system may play a role in the pathogenesis of SSc. However, further studies are required to address the exact role of CD163 in the pathogenesis of SSc and to determine whether it could help in the risk-stratification of the patients in this heterogeneous disease.

Disclosure of Interest: None declared

DOI: 10.1136/annrheumdis-2018-eular.2631
WEDNESDAY, 13 JUNE 2018

\section{A rollercoaster from bench to bedside and back again: paediatric rheumatology in the 21 st century}

\begin{tabular}{l|l}
\hline OP0096 & ADENO-ASSOCIATED VIRUS VECTOR-MEDIATED \\
INTERLEUKIN-10 INDUCTION PREVENTS VASCULAR \\
INFLAMMATION IN A MURINE MODEL OF KAWASAKI \\
DISEASE
\end{tabular}

J Nakamura $^{1,2}$, S. Watanabe ${ }^{1}$, H. Kimura ${ }^{1}$, H. Mizukami ${ }^{3}$, N. Nagi-Miura ${ }^{4}$, N. Ohno ${ }^{4}$, M. Takahashi ${ }^{1}$, S. Minota ${ }^{2} .{ }^{1}$ Division of Inflammation Research; ${ }^{2}$ Rheumatology and Clinical Immunology, ${ }^{3}$ Division of Genetic Therapeutics, Jichi Medical University, Shimotsuke, Tochigi; ${ }^{4}$ Laboratory for Immunopharmacology of Microbial Products, School of Pharmacy, Tokyo University of Pharmacy and Life Sciences, Hachioji, Tokyo, Japan

Background: Kawasaki disease (KD), which is a common paediatric heart disease, is characterised by coronary vasculitis and subsequently aneurysm formation. Although the administration of intravenous immunoglobulin (IVIG) is effective for reducing aneurysm formation, approximately $10 \%-20 \%$ of patients are resistant to this therapy. Therefore, additional therapeutic approaches for treating the IVIG-resistant patients need to be developed.

Candida albicans water-soluble fraction (CAWS)-induced vasculitis on coronary arteries and root of aorta is a frequently used murine model of KD. It has been considered that C-type lectin receptor Dectin-2 recognises CAWS. Recent studies showed CAWS-resistant strains of mice have higher serum IL-10 levels, which suggested that IL-10 might negatively regulate the development of CAWS induced vasculitis.

Objectives: The aim of the study is to investigate the therapeutic effect of IL-10 in CAWS-induced vasculitis and elucidate the underlying pathogenesis of KD.

Methods: To induce the expression of IL-10 in vivo, Adeno-associated virus (AAV) vectors encoding IL-10 were injected into DBA/2 mice. After the induction of IL-10, the mice were treated intraperitoneally with CAWS to induce vasculitis. Cardiac functions by echocardiography, inflammation and fibrosis by histological analyses, gene expression of inflammatory cytokines and fibrosis-related factors in the heart, and infiltrating cells by flow cytometry were assessed to evaluate the effects of IL- 10 .

For in vitro study, bone marrow-derived macrophages (BMDM) were stimulated with CAWS in presence or absence of IL-10. TNF- $\alpha$ and IL- 6 produced by the BMDM and Dectin-2 expressions on the BMDM were assessed.

Results: AAV-mediated induction of IL-10 significantly attenuated CAWSinduced cardiac functions (\%FS and LVEDD). Histological analyses revealed that $\mathrm{IL}-10$ markedly attenuated the vascular inflammation and fibrosis in the aortic root and coronary artery. Accordingly, increased gene expressions of inflammatory cytokines or fibrosis-related factors in the heart of CAWS-treated mice were significantly reduced by $\mathrm{IL}-10$. The predominant infiltrating inflammatory cells in vascular walls were Dectin- $2^{+} \mathrm{CD} 11 \mathrm{~b}^{+}$macrophages, and they were also decreased by IL-10.

Furthermore, we showed GM-CSF induced Dectin-2 expression on BMDM, and the GM-CSF-treated BMDM produced TNF- $\alpha$ and IL- 6 upon CAWS-stimulation. $\mathrm{IL}-10$ had no effect on the Dectin-2 expression but significantly inhibited the production of the cytokines. Finally, the AAV-mediated induction of IL-10 prevented the expression of TNF- $\alpha$ and IL- 6 in the heart of the mice treated with CAWS for 24 hours (at the early phase), but not GM-CSF and Dectin-2. These results suggest that GM-CSF mediates CAWS-induced vasculitis via Dectin-2 upregulation and IL-10 inhibits the downstream of GM-CSF and Dectin-2 signalling.

Conclusions: Our study has shown that IL-10 may have therapeutic application in the prevention of coronary vasculitis and aneurysm formation, and provided new insights into the mechanism underlying the pathogenesis of KD.

Disclosure of Interest: None declared

DOI: 10.1136/annrheumdis-2018-eular.2086

\section{OP0097 STIMULATED MONOCYTE-DERIVED MACROPHAGES FROM PATIENTS WITH ENTHESITIS RELATED ARTHRITIS SECRETE HIGHER LEVELS OF IL23 AND LOWER LEVELS OF INTERFERON GAMMA COMPARED TO HEALTHY CONTROLS}

C Fisher ${ }^{1,2}$, D. Eleftheriou ${ }^{1}$, D. Sen ${ }^{1,2}$, Y. Ioannou ${ }^{1} .{ }^{1}$ Arthritis Research UK Centre for Adolescent Rheumatology, University College London; ${ }^{2}$ National Institute for Health Research University College London Hospitals Biomedical Research Centre, University College London and University College London Hospital, London, UK

Background: Enthesitis related arthritis (ERA) is the subtype of juvenile idiopathic arthritis most closely related to adult spondyloarthropathy (SpA) 
Macrophages, the IL23/IL17 pathway and dysregulation of IFN $\gamma$ are strongly implicated in the pathogenesis of adult SpA but remain relatively unexplored in ERA. Objectives: To compare levels of IL23 and IFN $\gamma$ produced by monocyte-derived macrophages (MDMs) in the presence of lipopolysaccharide (LPS) from patients with ERA compared to healthy controls. The effect of clinical features such as enthesitis, peripheral and axial arthritis on IL23 and IFN $\gamma$ expression was also studied.

Methods: Peripheral blood monocytes from 39 patients $(68 \%$ HLA B27 positive, $84 \%$ male, median age 16 years 4 months, median disease duration 3 years 10 months) and 21 age and gender-matched healthy controls were differentiated in vitro with macrophage-colony stimulating factor in to macrophages. Differentiated cells were treated with IFN $\gamma$ for 24 hours to upregulate HLA B after which the cells were washed and stimulated with LPS (50 ng/mL). IL23 and IFN $\gamma$ expression from the cell culture supernatants were measured by ELISA and luminex assay respectively.

Results: IL23 expression was significantly higher in patients with ERA [median $53580 \mathrm{pg} / \mathrm{mL}$ (IQR $35735-83945 \mathrm{pg} / \mathrm{mL})$ VS $32110 \mathrm{pg} / \mathrm{mL}$ (IQR 13745$48235 \mathrm{pg} / \mathrm{mL}$ ), $\mathrm{p}=0.01$ ], particularly in patients who were HLA B27 positive [median $64650 \mathrm{pg} / \mathrm{mL}$ (IQR 36400-99650 pg/mL), p=0.0067]. IL23 was not significantly different between patients with and without peripheral arthritis or axial arthritis. However, patients who had active enthesitis (assessed clinically or on scan within 6 months of the sample) had significantly higher IL23 expression compared to patients without enthesitis [median 75905 pg/mL (IQR 36728-99425 pg/ $\mathrm{mL}$ vs $38485 \mathrm{pg} / \mathrm{mL}$ (IQR 29923-63725 pg/mL, $\mathrm{p}=0.014$ ] and healthy controls $[p=0.0017]$. Interestingly, patients who had both HLA B27 and active enthesitis had even higher levels of IL23 [median $79380 \mathrm{pg} / \mathrm{mL}$ (IQR 36540-103200 pg/ $\mathrm{mL}), \mathrm{p}=0.0018$ ]. Conversely, levels of IFN $\gamma$ were found to be lower from MDMs of patients with ERA compared to healthy controls, at baseline [median $3985 \mathrm{pg} / \mathrm{mL}$ (IQR 2820-6849 pg/mL) vs $6305 \mathrm{pg} / \mathrm{mL}$ (IQR 3938-8744 pg/mL), p=0.0054] and after LPS stimulation [median $9146 \mathrm{pg} / \mathrm{mL}$ (IQR $7438-11255 \mathrm{pg} / \mathrm{mL}$ ) vs $11693 \mathrm{pg} / \mathrm{mL}$ (IQR 9481-13435 pg/mL), $\mathrm{p}=0.013$ ]. No difference was found between patients who were HLA B27 positive and negative, although there was a trend towards lower levels of IFN $\gamma$ in patients with enthesitis, this was not statistically significant.

Conclusions: IL23 expression is significantly higher from stimulated MDMs of patients with ERA compared to healthy controls, especially in HLA B27 positive patients with active enthesitis. This suggests a role for IL23 in the pathogenesis of ERA and supports the hypothesis that this sub-population of patients might benefit from IL23 blockade. Interestingly, IFN $\gamma$ expression is lower in patients with ERA. Dysregulation of IFN $\gamma$ has been shown to cause upregulation of the IL23/17 pathway in animal models ${ }^{1}$ and thus may also contribute to the pathogenesis of ERA.

\section{REFERENCE:}

[1] Chu CQ, Swart D, Alcorn D, Tocker J, Elkon KB. Interferon-gamma regulates susceptibility to collagen-induced arthritis through suppression of interleukin-17. Arthritis Rheum 2007 Apr;56(4):1145-51.

Disclosure of Interest: None declared

DOI: 10.1136/annrheumdis-2018-eular.5377

\section{OP0098 CLINICAL PICTURE AND SPECTRUM OF CONDITIONS ASSOCIATED WITH NLRP12 GENE MUTATIONS}

M. Kostik ${ }^{1}$, E. Suspitsin ${ }^{1,2}$, A. Levina ${ }^{1}$, M. Guseva ${ }^{1}$, M. Dubko $^{1}$, L. Snegireva ${ }^{1}$, T. Likhacheva' ${ }^{1}$, A. Kazantseva ${ }^{1}$, M. Makhova ${ }^{1}$, A. Sokolenko ${ }^{1,2}$, E. Imyanitov $^{1,2}$, V. Chasnyk ${ }^{1} .{ }^{1}$ Saint-Petersburg State Pediatric Medical University, ${ }^{2}$ N.N. Petrov Research Institute of Oncology, Saint-Petersburg, Russian Federation

Background: NLRP12-related autoinflammatory disease (NLRP12-AID) is an exceptionally rare autosomal-dominant disorder caused by germ-line mutations in NLRP12 gene. Very few patients with NLRP12-AD have been identified worldwide, therefore there is a scarcity of data on phenotypic presentation of this syndrome.

Objectives: Here we provide evidence that NLRP12-AID may have clinical manifestations characteristic for primary immune deficiencies (PID).

Methods: 246 children with periodic fever (PF) of unknown origin were subjects to the next generation sequencing (NGS) analysis; 213 of these patients had signs of primary immunodeficiency (PID) manifested by recurrent infections, while 33 kids had isolated PF. The NGS panel was composed of 302 genes implicated in PID and/or AID.

Results: NLRP12 variants were detected in 20 cases (see the table 1). Median age of first AID-related fever episode was 12 months, ranging from 2 months to 13 years. Increased association with immune-mediated disease $(n=15,75 \%)$ was observed. Nine patients demonstrated increased susceptibility to infection and two children suffered from Crohn's disease. Administration of short courses of
NSAID or corticosteroids resulted in resolution of the disease flare. In one severe case canakinumab (anti-interleukine-1 $\beta$ antibody) was successfully used.

Abstract OP0098 - Table 1

\begin{tabular}{|c|c|c|c|}
\hline $\begin{array}{l}\text { NLRP12 } \\
\text { mutation }\end{array}$ & ID, sex & NLRP12 related symptoms & Comorbid disease \\
\hline p.H304Y & $\begin{array}{l}\text { PK, f } \\
\text { RI, m } \\
\text { AA, f }\end{array}$ & $\begin{array}{c}\text { PF, } 1 \mathrm{~d} / \text { week } \\
\text { Cyclic vomiting with hCRP since } \\
\text { newborn Permanent aphtous } \\
\text { stomatitis, PFAPA, AP }\end{array}$ & $\begin{array}{c}\text { CVID, AlHA, pancytopenia, } \\
\text { S, ILD Severe CD, food and } \\
\text { drug allergy }\end{array}$ \\
\hline Asn715>Ser & $\begin{array}{l}\text { ZV, m } \\
\text { KA, m } \\
\text { VM, m }\end{array}$ & $\begin{array}{l}\text { PF (infections?) PF } 5-7 d / \text { month } \\
\text { with LA, redness, eye pain }\end{array}$ & $\begin{array}{c}\text { Congenital red-cell aplasia, } \\
\text { AIHA, S, LA } \\
\text { CVID, AIHA, S, LA, ILD, } \\
\text { thrombocytopenia } \\
\text { Chronic posterior scleritis }\end{array}$ \\
\hline p.F402L & $\begin{array}{l}\mathrm{GA}, \mathrm{f} \\
\mathrm{GE}, \mathrm{m}\end{array}$ & $\begin{array}{l}\text { PF } \\
\text { PF }\end{array}$ & $\begin{array}{l}\text { Episodes of severe } \\
\text { respiratory infection Severe } \\
\text { CD, S }\end{array}$ \\
\hline Thr861Ser & $\begin{array}{l}\mathrm{CA}, \mathrm{m} \\
\mathrm{KS}, \mathrm{m}\end{array}$ & $\begin{array}{c}\text { PF (infections) } \\
\text { Episodic AP, diarrhoea }\end{array}$ & $\begin{array}{l}\text { Meningitis, septic shock, } \\
\text { otitis, }\end{array}$ \\
\hline Gln417Leu & $\begin{array}{l}\text { AR, } m \\
\text { SK, } m\end{array}$ & $\begin{array}{c}1 \mathrm{~d} \text { PF } \\
\text { Persistent fever }\end{array}$ & Severe recurrent MAS, ILD \\
\hline Arg352Cys & $D E, f$ & $\begin{array}{l}\text { PF, S, A, AP, urticaria, growth } \\
\text { delay, }\end{array}$ & \\
\hline p.Ala57Ser & $\mathrm{AK}, \mathrm{m}$ & $\begin{array}{c}\text { Respiratory and intestinal infections } \\
\text { with PF }\end{array}$ & Bacterial meningitis \\
\hline p.Ala5Glu & $\mathrm{HT}, \mathrm{m}$ & $\begin{array}{l}\text { PFAPA-like syndrome headackes } \\
\text { +eye pain }\end{array}$ & \\
\hline p.Arg723Gln & $B A, f$ & $\mathrm{PF}, \mathrm{A}$ & \\
\hline p.Val936Leu & $G A, f$ & PF (infections?) & $\begin{array}{l}\text { Recurrent purulent sinusitis } \\
\text { wlo fever }\end{array}$ \\
\hline $\begin{array}{l}\text { p.Tyr246Cys } \\
\text { p.Gly52Ser }\end{array}$ & $\begin{array}{l}E S, f \\
K V, f\end{array}$ & $\begin{array}{l}\text { FCAS, relapsed aseptic peritonitis } \\
\text { PF (infections?) }\end{array}$ & \\
\hline p.R738fs & $\mathrm{SD}, \mathrm{m}$ & $\begin{array}{l}\text { 1-4d of PF, cold-induced, 3-4/ } \\
\text { year, }\end{array}$ & $\begin{array}{c}\text { pneumonia, febrile seizures, } \\
\text { brain oedema }\end{array}$ \\
\hline
\end{tabular}

Abbreviations: A-arthralgia, ABA-abatacept, AlHA-autoimmune hemolytic anaemia, AP-abdominal pain, CD-Crohn's disease, CKB-canakinumab, CVID-common variable immunodeficiency, d-day, ILD-interstitial lung disease, LAlymphadenopathy, PF-periodic fever, S-splenomegaly

Conclusions: Significant number of patients with genetically assigned diagnosis of NLPR12-AID have clinical features which close resemble PID. This phenotypic overlap may result in underdiagnosis of NLPR12-AID among patients with PID.

Acknowledgements: This work has been supported by Russian Foundation for Basic Research (grant number RFBR 16-04-00 159a).

Disclosure of Interest: None declared

DOI: 10.1136/annrheumdis-2018-eular.5650

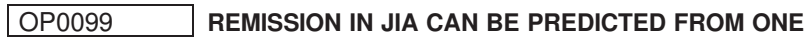 YEAR FOLLOWING DIAGNOSIS IN A UK MULTICENTRE INCEPTION COHORT}

S.J.W Shoop-Worrall ${ }^{1,2}$, S.M.M. Verstappen ${ }^{1,2}$, J.E. McDonagh $^{1,2}$, W. Thomson $^{1,2}$, K.L. Hyrich ${ }^{1,2}$, on behalf of CAPS. ${ }^{1}$ The University of Manchester, ${ }^{2}$ Manchester Foundation Trust, Manchester, UK

Background: One of the primary aims for children and young people with JIA is remission. Early improvement in disease likely predicts greater chance of remission in the longer-term.

Objectives: To assess the association between change in disease activity over the first year of disease and later occurrence of remission.

Methods: Children and young people with oligoarticular, RF-negative or RF-positive polyarticular JIA recruited to the Childhood Arthritis Prospective Study prior to January 2014 were selected for analysis.

Two definitions of remission based on clinically inactive disease at two consecu tive study follow-ups were explored: 1) Wallace's preliminary criteria and 2) the clinical Juvenile Arthritis Disease Activity Score in 10 joints (cJADAS10). Associa tion between changes in JIA core outcome variables and pain over the first year of disease and later occurrence of remission were analysed using logistic regression models, adjusting additionally for age, gender, symptom duration and predictor value at presentation. Oligoarticular and polyarticular JIA were modelled separately. Multiple imputation accounted for missing data.

Results: A total of 1045 children were included. Of these, $70 \%$ were female and the majority had oligoarticular JIA (67\%). The median age at presentation was 7 years (IQR 3,11$)$. Within three years, $25 \%$ achieved remission according to Wallace's criteria and 39\% according to cJADAS10. More children with oligoarticular JIA achieved remission than those with polyarticular JIA (table 1). 